\title{
Dose Expansion Cohort
}

National Cancer Institute

\section{Source}

National Cancer Institute. Dose Expansion Cohort. NCI Thesaurus. Code C133188.

A group of subjects in a phase I trial, usually accrued after the maximum tolerated dose maximum tolerated dose has been established. These subjects, often with additional eligibility criteria, are used to: confirm that a safe level of drug exposure has been established, obtain preliminary evidence of efficacy, and identify specific patient subgroups that might derive particular benefits from the investig ational treatment. 